\title{
Ultrasonographic evaluation of fetal sacral length and correlation with gestational age. Quader S A ${ }^{1}$, Islam M S ${ }^{2}$, Islam M M ${ }^{3}$, Hossain M Z ${ }^{4}$
}

1. Major Dr. Syed Abdul Quader, Classified Specialist Radiology \& Imaging, Combined Military Hospital, Bogra, Bangladesh. 2. Dr. Md. Saiful Islam, Assistant Professor, Department of Radiology \& Imaging, Jahurul Islam Medical College \& Hospital, Kishoregonj, Bangladesh. 3. Dr. Md. Menhazul Islam, Medical Officer, Department of Radiology \& Imaging, Bangabandhu Sheikh Mujib Medical University, Dhaka, Bangladesh. 4. Dr. Md. Zakir Hossain, Assistant Professor, Department of Orthopedics, Jahurul Islam Medical College \& Hospital, Kishoregonj, Bangladesh

Article info: Received: April 10, 2016, Accepted: May 10, 2016,

\section{Abstract}

Background: During the last three decades, ultrasonographics of a number of fetal osseous structures have been adopted for the assessment of gestational age and the evaluation of fetal growth and development. The most frequently used morphometric parameters include biparietal diameter, head circumference and femur length. Moreover, nomograms for other osseous structures, such as mandible, clavicle, scapula, vertebral arch, iliac bone and foot length have been established. Another suggestion is to measure the length of fetal sacrum, which should be visualized as a part of routine ultrasonographic evaluation of a fetus. Hence, associated congenital anomalies such as sacral agenesis or sacrococcygeal teratoma can be detected simultaneously .Objective: To evaluate the usefulness of fetal sacral length in determination of gestational age at 15th to 40th weeks of pregnancy. Method: This study was carried out on 441 normal pregnant women between 15 to 40 weeks of gestation over a period of 24 months to find out the correlation between gestational ages with fetal sacral length measured by ultrasonographically. 41 patients were excluded from my study as 09 had multiple pregnancies, 02 had fetal congenital anomaly; out of them one had anencephaly and one had lumbo-sacral meningocele and complete visualization of sacrum was not possible for 30 fetuses. Result: Measurements of fetal sacral length were performed by utilizing gray scale real time ultrasound scanner equipped with $3.5 \mathrm{MHz}$ convex transducer and gestational age was estimated by LMP.The mean gestational age was 27.52 with standard deviation of mean $( \pm \mathrm{SD})$ was \pm 7.28 weeks and gestational age ranging from 15 to 40 weeks. $40.0 \%$ percent were Primigravida, $31.75 \%$ percent were $2^{\text {nd }}$ gravida, 18.25 percent were $3^{\text {rd }}$ gravida, 7.75 percent were $4^{\text {th }}$ gravid and $1.75 \%$ percent were $5^{\text {th }}$ and more gravida. Most of the cases were Primigravida and $2^{\text {nd }}$ gravida. A positive significant correlation were found between fetal sacral length with gestational age (wk) $\left(\mathrm{r}=0.998 ; \mathrm{p}<0.001\right.$ and $\left.\mathrm{R}^{2}=99 \%\right)$

\section{*Corresponding Author:}

\section{Dr. Md. Menhazul Islam}

Medical Officer

Department of Radiology \& Imaging,

Bangabandhu Sheikh Mujib Medical University,

Dhaka, Bangladesh

E-mail: ahnafbadal@gmail.com

Cell: +88 01822971734
Conclusion:In this study it is observed that there is significant correlation of fetal sacral length with estimated fetal age so, it can be concluded in the present study that fetal sacral length(FSL) can be used as a parameter for estimation of gestational age. 


\section{Introduction}

A healthy new born is the goal of every expectant mother and her obstetrician. As the clinical estimation of gestational age to assess fetal growth is not reliable, prenatal ultrasonography provides an opportunity to more accurately assess fetal growth. Ultrasound biometry of the fetus is now the gold standard for assessing the fetal growth ${ }^{1}$

The most frequently used morphometric parameters include biparietal diameter, head circumference and femur length. Moreover, nomograms for other osseous structures, such as mandible, clavicle, scapula, vertebral arch, iliac bone and foot length have been established ${ }^{2}$. Another suggestion is to measure the length of fetal sacrum, which should be visualized as a part of routine ultrasonographic evaluation of a fetus. Hence, associated congenital anomalies such as sacral agenesis or sacrococcygealteratoma can be detected simultaneously ${ }^{3}$.

The fetal sacral length was determined according to the method described by ${ }^{3}$. Therefore, the distance between the distal tip of spine (namely fifth sacral vertebra) and anterior superior aspect of the first sacral vertebra was measured by calipers at the sagittal plane. It is well known that coccyx is yet ossified in the third trimester of pregnancy during which this cartilaginous structure is visualized as a thin echogenic plate at the distal age of the central ossification center of fifth sacral vertebra. This way care is maximized to exclude this echogenic plate when fetal sacral length was measured in third trimester of pregnancies ${ }^{2}$. This study was done with the nomogram produced by $^{2}$ and find out its accuracy in the determination of gestational age by using real time ultrasound in Bangladeshi population.

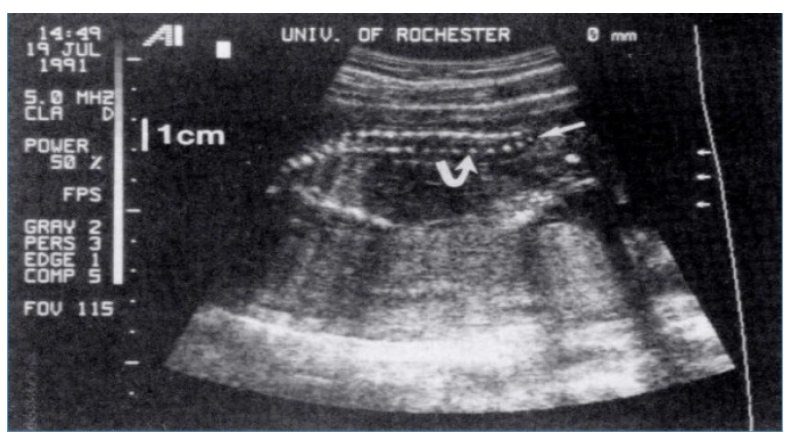

Figure 1.Ultrasonographic scan of sacral length, obtained from breech presenting fetus at 18 weeks 2 days of gestation, measuring $1.8 \mathrm{~cm}$. Curved arrow, Proximal landmark (anterosuperior margin of $\quad$ S1 or sacral promontory);straight arrow, distal landmark (anterorinferior tip of S5). 


\section{Materials and methods :}

This study was carried out on 441 normal pregnant women between 15 to 40 weeks of gestation referred to the department of radiology and imaging of $\mathrm{MMCH}$ for ultrasonic evaluation of pregnancy profile.

All the subjects were properly informed about the study and written consent was taken from them. Then a longitudinal scan was carried out to determine the position of fetal spine. The probe follows the caudal spine till it is traced completely. The length of last five sacral vertebrae was measured in millimeter by electronic calipers. The comparison was made between the fetal sacral length and the fetal gestational age.

Gestational age was determined on the basis of LMP than fetal sacral length was measured by sonographically. Then the relevant data were collected in a master table. Data from the master table were imputed in the computer. Statistical analysis was performed using the software package SPSS for Windows, version 17.0 (SPSS Inc., Chicago, IL, USA). Then a correlation was established between gestational age and fetal sacral length. Then data were presented by table and graphs.

41 patients were excluded from my study as 09 had multiple pregnancies, 02 had fetal congenital anomaly; out of them one had anencephaly and one had lumbo-sacral meningocele and complete visualization of sacrum was not possible for 30 fetuses.

Twenty six groups were studied, each group having $15(+/-5)$ patients with all 5 to 10 patients in the same gestational period, from 15 to 40 weeks.

\section{Results}

This cross sectional study was carried out during the study period of 24 months on 400 healthy pregnant women. During this study, gestational age was determined by calculating the first day of last menstrual period (LMP)). Fetal sacral lengths (FSL) were measured by two-dimensional sonography and determinant in different gestational age ranging from 15 to 40 weeks and were correlated with gestational age according to LMP.

Results of the study and statistical analysis were presented by tables, figures, graphs, diagrams, charts, photographs etc.

\section{Gravida of the pregnant women}

In this study out of 400 subjects, 40.0 percent were Primigravida, 31.75 percent were $2^{\text {nd }}$ gravida, 18.25 percent were $3^{\text {rd }}$ gravida, 7.75 percent were $4^{\text {th }}$ gravid and 1.75 percent were $5^{\text {th }}$ and more gravida. Most of the cases were Primigravida and $2^{\text {nd }}$ 
gravid. The mean gravida of the subjects was 1.99 with standard deviation of mean $( \pm \mathrm{SD})$ was \pm 1.03 among total subject (Table-II) (Figure-5).

Table-II: Distribution of the study subjects according to gravida $(n=400)$.

\begin{tabular}{|l|l|l|}
\hline Gravida & Number of patient & Percentage \\
\hline Primi & 160 & $40 \%$ \\
\hline $2^{\text {nd }}$ & 127 & $31.75 \%$ \\
\hline $3^{\text {rd }}$ & 73 & $18.25 \%$ \\
\hline $4^{\text {th }}$ & 31 & $7.75 \%$ \\
\hline$\geq 5^{\text {th }}$ & 9 & $1.75 \%$ \\
\hline Mean \pm SD $1.9925 \pm 1.0393 R$ Range & $(1-5)$ \\
\hline
\end{tabular}

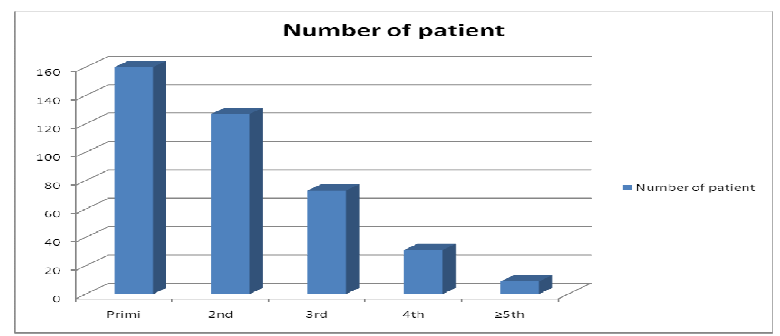

Figure-5: Bar diagram showing distribution of study subject according to gravida $(n=400)$.

Correlation between gestational age and fetal sacral length of pregnant woman $(n=400)$
Gestational age was expressed in weeks and ultrasonographically measured fetal sacral length in $\mathrm{mm}$ show significant positive correlation in between them.

The pearson' correlation test showed positive correlation between the dependent fetal sacral length (in mm) and independent gestational age (in weeks). The relation between fetal sacral length and gestational age was statistically significant $(r=0.998$, $\mathrm{p}=.001)$

A regression analysis was also done. The coefficient of determination $\left(\mathrm{R}^{2}\right)$ was found $99 \%$.

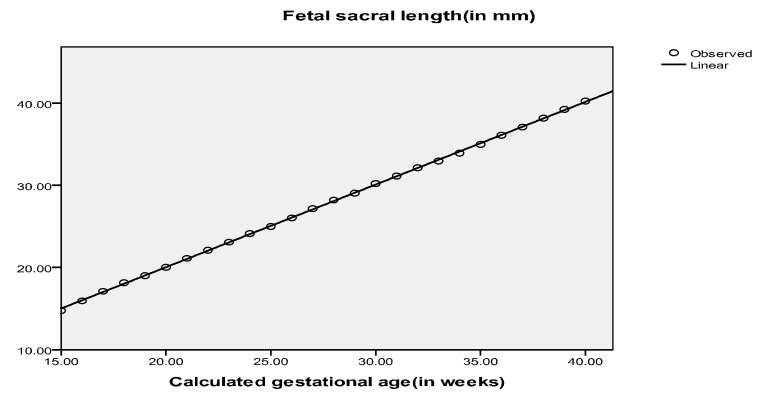

Figure-6: Scatter diagram showing the positive correlation $(r=0.998 ; p<0.001)$ between gestation age (wk) with fetal sacral length (mm). 
Table-III: Distribution of percentile,

Mean, Standard Deviation, Median, Mode minimum and maximum value of

fetal sacral length according to gestational age at different weeks of gestation $(n=400)$.

\begin{tabular}{|c|c|c|c|c|c|c|c|c|c|c|}
\hline \multirow{2}{*}{$\begin{array}{c}\text { GA. } \\
\text { age } \\
\text { (in } \\
\text { week } \\
\text { s) }\end{array}$} & \multirow[t]{2}{*}{$\mathbf{N}$} & \multicolumn{9}{|c|}{ Fetal sacral length(mm) } \\
\hline & & P5 & P50 & $\begin{array}{l}\text { P9 } \\
5\end{array}$ & $\begin{array}{l}\text { Me } \\
\text { an }\end{array}$ & SD & $\begin{array}{c}\text { Mod } \\
\text { e }\end{array}$ & $\underset{\mathbf{n}}{\text { Media }}$ & $\begin{array}{l}\text { Mi } \\
\text { n }\end{array}$ & Max \\
\hline 15 & $\begin{array}{l}1 \\
3\end{array}$ & $\begin{array}{l}14 . \\
1\end{array}$ & $\begin{array}{l}14 . \\
9\end{array}$ & $\begin{array}{l}15 . \\
5\end{array}$ & $\begin{array}{l}14.7 \\
6\end{array}$ & $\begin{array}{l}0.48 \\
05\end{array}$ & $\begin{array}{l}14 . \\
2 *\end{array}$ & $\begin{array}{l}14 . \\
9\end{array}$ & $\begin{array}{l}14 . \\
1\end{array}$ & $\begin{array}{l}15 . \\
5\end{array}$ \\
\hline 16 & $\begin{array}{l}1 \\
6\end{array}$ & $\begin{array}{l}14 . \\
9\end{array}$ & $\begin{array}{l}15 . \\
9\end{array}$ & $\begin{array}{l}16 . \\
8\end{array}$ & $\begin{array}{l}15.9 \\
4\end{array}$ & $\begin{array}{l}0.48 \\
16\end{array}$ & $\begin{array}{l}15 . \\
9\end{array}$ & $\begin{array}{l}15 . \\
9\end{array}$ & $\begin{array}{l}14 . \\
9\end{array}$ & $\begin{array}{l}16 . \\
8\end{array}$ \\
\hline 17 & $\begin{array}{l}1 \\
6 \\
\end{array}$ & $\begin{array}{l}16 . \\
7\end{array}$ & $\begin{array}{l}17 . \\
15\end{array}$ & $\begin{array}{l}17 . \\
5\end{array}$ & $\begin{array}{l}17.1 \\
1\end{array}$ & $\begin{array}{l}0.29 \\
86 \\
\end{array}$ & $\begin{array}{l}16 . \\
8\end{array}$ & $\begin{array}{l}17 . \\
15\end{array}$ & $\begin{array}{l}16 . \\
7\end{array}$ & $\begin{array}{l}17 . \\
5\end{array}$ \\
\hline 18 & $\begin{array}{l}1 \\
2 \\
\end{array}$ & $\begin{array}{l}17 . \\
6\end{array}$ & $\begin{array}{l}18 . \\
2\end{array}$ & $\begin{array}{l}18 . \\
9\end{array}$ & $\begin{array}{l}18.1 \\
6 \\
\end{array}$ & $\begin{array}{ll}0.38 \\
21 \\
\end{array}$ & $\begin{array}{l}18 . \\
2 *\end{array}$ & $\begin{array}{l}18 . \\
2\end{array}$ & $\begin{array}{l}17 . \\
6\end{array}$ & $\begin{array}{l}18 . \\
9\end{array}$ \\
\hline 19 & $\begin{array}{l}1 \\
7\end{array}$ & $\begin{array}{l}18 . \\
5\end{array}$ & $\begin{array}{l}19 . \\
0\end{array}$ & $\begin{array}{l}19 . \\
5\end{array}$ & $\begin{array}{l}19.0 \\
2\end{array}$ & $\begin{array}{l}0.33 \\
45 \\
\end{array}$ & $\begin{array}{l}18 . \\
8^{*}\end{array}$ & $\begin{array}{l}19 . \\
\text { 0 }\end{array}$ & $\begin{array}{l}18 . \\
5\end{array}$ & $\begin{array}{l}19 . \\
5\end{array}$ \\
\hline 20 & $\begin{array}{l}1 \\
8 \\
\end{array}$ & $\begin{array}{l}19 . \\
5\end{array}$ & $\begin{array}{l}20 . \\
05\end{array}$ & $\begin{array}{l}20 . \\
8\end{array}$ & $\begin{array}{l}20.0 \\
2\end{array}$ & $\begin{array}{l}0.32 \\
86 \\
\end{array}$ & $\begin{array}{l}20 . \\
2\end{array}$ & $\begin{array}{l}20 . \\
05\end{array}$ & $\begin{array}{l}19 . \\
5\end{array}$ & $\begin{array}{l}20 . \\
8 \\
\end{array}$ \\
\hline 21 & $\begin{array}{l}1 \\
4 \\
\end{array}$ & $\begin{array}{l}20 . \\
6 \\
\end{array}$ & $\begin{array}{l}21 . \\
25 \\
\end{array}$ & $\begin{array}{l}21 . \\
5\end{array}$ & $\begin{array}{l}21.1 \\
2 \\
\end{array}$ & \begin{tabular}{|l|}
0.31 \\
17 \\
\end{tabular} & $\begin{array}{l}21 . \\
3\end{array}$ & $\begin{array}{l}21 . \\
25\end{array}$ & $\begin{array}{l}20 . \\
6 \\
\end{array}$ & $\begin{array}{l}21 . \\
5\end{array}$ \\
\hline 22 & $\begin{array}{l}1 \\
1 \\
\end{array}$ & $\begin{array}{l}21 . \\
6 \\
\end{array}$ & $\begin{array}{l}22 . \\
1 \\
\end{array}$ & $\begin{array}{l}22 . \\
6 \\
\end{array}$ & $\begin{array}{l}22.1 \\
2 \\
\end{array}$ & \begin{tabular}{|l|}
0.34 \\
87 \\
\end{tabular} & $\begin{array}{l}22 . \\
5 \\
\end{array}$ & $\begin{array}{l}22 . \\
1 \\
\end{array}$ & $\begin{array}{l}21 . \\
6 \\
\end{array}$ & $\begin{array}{l}22 . \\
6 \\
\end{array}$ \\
\hline 23 & $\begin{array}{l}1 \\
1 \\
\end{array}$ & $\begin{array}{l}22 . \\
6 \\
\end{array}$ & $\begin{array}{l}23 . \\
1 \\
\end{array}$ & $\begin{array}{l}23 . \\
5\end{array}$ & $\begin{array}{l}23.0 \\
9 \\
\end{array}$ & \begin{tabular}{|l|}
0.29 \\
81 \\
\end{tabular} & $\begin{array}{l}23 . \\
1\end{array}$ & $\begin{array}{l}23 . \\
1\end{array}$ & $\begin{array}{l}22 . \\
6 \\
\end{array}$ & $\begin{array}{l}23 . \\
5\end{array}$ \\
\hline 24 & $\begin{array}{l}1 \\
3 \\
\end{array}$ & $\begin{array}{l}23 . \\
6\end{array}$ & $\begin{array}{l}24 . \\
2\end{array}$ & $\begin{array}{l}24 . \\
5\end{array}$ & $\begin{array}{l}24.1 \\
3 \\
\end{array}$ & $\begin{array}{l}0.32 \\
02 \\
\end{array}$ & $\begin{array}{l}24 . \\
5\end{array}$ & $\begin{array}{l}24 . \\
2\end{array}$ & $\begin{array}{l}23 . \\
6\end{array}$ & $\begin{array}{l}24 . \\
5\end{array}$ \\
\hline 25 & $\begin{array}{l}1 \\
9 \\
\end{array}$ & $\begin{array}{l}24 . \\
5\end{array}$ & $\begin{array}{l}25 . \\
0 \\
\end{array}$ & $\begin{array}{l}25 . \\
6 \\
\end{array}$ & $\begin{array}{l}25.0 \\
1 \\
\end{array}$ & $\begin{array}{l}0.27 \\
05 \\
\end{array}$ & $\begin{array}{l}24 . \\
8 \\
\end{array}$ & $\begin{array}{l}25 . \\
0 \\
\end{array}$ & $\begin{array}{l}24 . \\
5 \\
\end{array}$ & $\begin{array}{l}25 . \\
6 \\
\end{array}$ \\
\hline 26 & $\begin{array}{l}1 \\
7 \\
\end{array}$ & $\begin{array}{l}25 . \\
5 \\
\end{array}$ & $\begin{array}{l}26 . \\
0\end{array}$ & $\begin{array}{l}26 . \\
5 \\
\end{array}$ & $\begin{array}{l}26.0 \\
4 \\
\end{array}$ & $\begin{array}{l}0.28 \\
95 \\
\end{array}$ & $\begin{array}{l}26 . \\
\text { 0 }\end{array}$ & $\begin{array}{l}26 . \\
\text { 0 }\end{array}$ & $\begin{array}{l}25 . \\
5 \\
\end{array}$ & $\begin{array}{l}26 . \\
5\end{array}$ \\
\hline 27 & $\begin{array}{l}1 \\
7 \\
\end{array}$ & $\begin{array}{l}26 . \\
5 \\
\end{array}$ & $\begin{array}{l}27 . \\
2 \\
\end{array}$ & $\begin{array}{l}27 . \\
6\end{array}$ & $\begin{array}{l}27.1 \\
6 \\
\end{array}$ & $\begin{array}{l}0.30 \\
81 \\
\end{array}$ & $\begin{array}{l}27 . \\
4 \\
\end{array}$ & $\begin{array}{l}27 . \\
2 \\
\end{array}$ & $\begin{array}{l}26 . \\
5 \\
\end{array}$ & $\begin{array}{l}27 . \\
6 \\
\end{array}$ \\
\hline 28 & $\begin{array}{l}1 \\
7 \\
\end{array}$ & $\begin{array}{l}27 . \\
7\end{array}$ & $\begin{array}{l}28 . \\
3 \\
\end{array}$ & $\begin{array}{l}28 . \\
7 \\
\end{array}$ & $\begin{array}{l}28.2 \\
2 \\
\end{array}$ & $\begin{array}{l}0.37 \\
54 \\
\end{array}$ & $\begin{array}{l}28 . \\
6 \\
\end{array}$ & $\begin{array}{l}28 . \\
3 \\
\end{array}$ & $\begin{array}{l}27 . \\
7\end{array}$ & $\begin{array}{l}28 . \\
7\end{array}$ \\
\hline 29 & $\begin{array}{l}2 \\
3 \\
\end{array}$ & $\begin{array}{l}28 . \\
5\end{array}$ & $\begin{array}{l}28 . \\
9 \\
\end{array}$ & $\begin{array}{l}29 . \\
6\end{array}$ & $\begin{array}{l}29.0 \\
5\end{array}$ & $\begin{array}{l}0.38 \\
83 \\
\end{array}$ & $\begin{array}{l}28 . \\
8\end{array}$ & $\begin{array}{l}28 . \\
9\end{array}$ & $\begin{array}{l}28 . \\
5\end{array}$ & $\begin{array}{l}29 . \\
6\end{array}$ \\
\hline 30 & $\begin{array}{l}1 \\
6 \\
\end{array}$ & $\begin{array}{l}29 . \\
3 \\
\end{array}$ & $\begin{array}{l}30 . \\
3 \\
\end{array}$ & $\begin{array}{l}30 . \\
7 \\
\end{array}$ & $\begin{array}{l}30.2 \\
1 \\
\end{array}$ & $\begin{array}{l}0.36 \\
91 \\
\end{array}$ & $\begin{array}{l}30 . \\
3 \\
\end{array}$ & $\begin{array}{l}30 . \\
3 \\
\end{array}$ & $\begin{array}{l}29 . \\
3 \\
\end{array}$ & $\begin{array}{l}30 . \\
7 \\
\end{array}$ \\
\hline 31 & $\begin{array}{l}1 \\
8 \\
\end{array}$ & $\begin{array}{l}30 . \\
7 \\
\end{array}$ & $\begin{array}{l}31 . \\
15 \\
\end{array}$ & $\begin{array}{l}31 . \\
7\end{array}$ & $\begin{array}{l}31.1 \\
3 \\
\end{array}$ & $\begin{array}{ll}0.27 \\
65 \\
\end{array}$ & $\begin{array}{l}31 . \\
4\end{array}$ & $\begin{array}{l}31 . \\
15\end{array}$ & $\begin{array}{l}30 . \\
7 \\
\end{array}$ & $\begin{array}{l}31 . \\
7\end{array}$ \\
\hline 32 & $\begin{array}{l}1 \\
7 \\
\end{array}$ & $\begin{array}{l}31 . \\
5\end{array}$ & $\begin{array}{l}32 . \\
2\end{array}$ & $\begin{array}{l}32 . \\
6\end{array}$ & $\begin{array}{l}32.1 \\
4 \\
\end{array}$ & $\begin{array}{l}0.34 \\
47 \\
\end{array}$ & $\begin{array}{l}32 . \\
5\end{array}$ & $\begin{array}{l}32 . \\
2\end{array}$ & $\begin{array}{l}31 . \\
5\end{array}$ & $\begin{array}{l}32 . \\
6\end{array}$ \\
\hline 33 & $\begin{array}{l}1 \\
7 \\
\end{array}$ & $\begin{array}{l}32 . \\
5\end{array}$ & $\begin{array}{l}32 . \\
9 \\
\end{array}$ & $\begin{array}{l}33 . \\
5\end{array}$ & $\begin{array}{l}32.9 \\
8 \\
\end{array}$ & $\begin{array}{l}0.28 \\
91 \\
\end{array}$ & $\begin{array}{l}32 . \\
9 \\
\end{array}$ & $\begin{array}{l}32 . \\
9 \\
\end{array}$ & $\begin{array}{l}32 . \\
5\end{array}$ & $\begin{array}{l}\text { 33. } \\
\mathbf{5}\end{array}$ \\
\hline 34 & $\begin{array}{l}1 \\
7 \\
\end{array}$ & $\begin{array}{l}33 . \\
5 \\
\end{array}$ & $\begin{array}{l}33 . \\
9 \\
\end{array}$ & $\begin{array}{l}34 . \\
4 \\
\end{array}$ & $\begin{array}{l}33.9 \\
4 \\
\end{array}$ & $\begin{array}{l}0.30 \\
23 \\
\end{array}$ & $\begin{array}{l}33 . \\
9 \\
\end{array}$ & $\begin{array}{l}33 . \\
9 \\
\end{array}$ & $\begin{array}{l}33 . \\
5 \\
\end{array}$ & $\begin{array}{l}34 . \\
4 \\
\end{array}$ \\
\hline 35 & $\begin{array}{l}1 \\
6 \\
\end{array}$ & $\begin{array}{l}34 . \\
6 \\
\end{array}$ & $\begin{array}{l}35 . \\
05\end{array}$ & $\begin{array}{l}35 . \\
5 \\
\end{array}$ & $\begin{array}{l}\text { 35.0 } \\
\text { 0 } \\
\end{array}$ & $\begin{array}{ll}0.31 \\
83 \\
\end{array}$ & $\begin{array}{l}34 . \\
6 \\
\end{array}$ & $\begin{array}{l}35 . \\
\mathbf{0 5}\end{array}$ & $\begin{array}{l}34 . \\
6 \\
\end{array}$ & $\begin{array}{l}35 . \\
5\end{array}$ \\
\hline 36 & $\begin{array}{l}1 \\
1\end{array}$ & $\begin{array}{l}35 . \\
7\end{array}$ & $\begin{array}{l}36 . \\
\text { 0 } \\
\end{array}$ & $\begin{array}{l}36 . \\
8 \\
\end{array}$ & $\begin{array}{l}36.1 \\
0 \\
\end{array}$ & $\begin{array}{l}0.39 \\
10\end{array}$ & $\begin{array}{l}35 \\
7 *\end{array}$ & $\begin{array}{l}36 . \\
\text { 0 }\end{array}$ & $\begin{array}{l}35 . \\
7\end{array}$ & $\begin{array}{l}36 . \\
8 \\
\end{array}$ \\
\hline 37 & $\begin{array}{l}1 \\
2 \\
\end{array}$ & $\begin{array}{l}36 . \\
5 \\
\end{array}$ & $\begin{array}{l}37 . \\
1\end{array}$ & $\begin{array}{l}37 . \\
8\end{array}$ & $\begin{array}{l}37.1 \\
0 \\
\end{array}$ & $\begin{array}{l}0.41 \\
00 \\
\end{array}$ & $\begin{array}{l}37 . \\
1\end{array}$ & $\begin{array}{l}37 . \\
1\end{array}$ & $\begin{array}{l}36 . \\
5\end{array}$ & $\begin{array}{l}37 . \\
8\end{array}$ \\
\hline 38 & $\begin{array}{l}\mathbf{1} \\
\mathbf{0} \\
\end{array}$ & $\begin{array}{l}37 . \\
8 \\
\end{array}$ & $\begin{array}{l}38 . \\
1 \\
\end{array}$ & $\begin{array}{l}38 . \\
7 \\
\end{array}$ & $\begin{array}{l}38.1 \\
8 \\
\end{array}$ & $\begin{array}{l}0.31 \\
19 \\
\end{array}$ & $\begin{array}{l}37 . \\
\text { 9* }\end{array}$ & $\begin{array}{l}38 . \\
1 \\
\end{array}$ & $\begin{array}{l}37 . \\
8 \\
\end{array}$ & $\begin{array}{l}38 . \\
7 \\
\end{array}$ \\
\hline 39 & $\begin{array}{l}1 \\
5 \\
\end{array}$ & $\begin{array}{l}38 . \\
7 \\
\end{array}$ & $\begin{array}{l}39 . \\
3 \\
\end{array}$ & $\begin{array}{l}39 . \\
6 \\
\end{array}$ & $\begin{array}{l}39.2 \\
4 \\
\end{array}$ & \begin{tabular}{|l|}
0.29 \\
47 \\
\end{tabular} & $\begin{array}{l}39 . \\
3\end{array}$ & $\begin{array}{l}39 . \\
3\end{array}$ & $\begin{array}{l}38 . \\
7 \\
\end{array}$ & $\begin{array}{l}39 . \\
6\end{array}$ \\
\hline 40 & $\begin{array}{l}1 \\
7 \\
\end{array}$ & $\begin{array}{l}39 . \\
4\end{array}$ & $\begin{array}{l}40 . \\
1\end{array}$ & $\begin{array}{l}41 . \\
9\end{array}$ & $\begin{array}{l}40.2 \\
6 \\
\end{array}$ & $\begin{array}{l}0.67 \\
44 \\
\end{array}$ & $\begin{array}{l}40 . \\
4\end{array}$ & $\begin{array}{l}40 . \\
1\end{array}$ & $\begin{array}{l}39 . \\
4\end{array}$ & $\begin{array}{l}41 . \\
9\end{array}$ \\
\hline
\end{tabular}

*Multiple modes exist. The smallest value is shown.

\section{Discussion}

The practice of assessing gestational age in early gestation is valuable in detection of growth aberration in later stages of pregnancy. In addition, fetal biometry distinguishes the normal from abnormal fetal structures. It is therefore important that fetal biometry be performed for local population and local charts of normal biometry be constructed and followed for these populations and ethnic groups. The standard of fetal ultrasound biometry was started after $^{4}$ published, probably the first paper, on fetal ultrasound cephalometery in 1964.

The fetal sacral length increases in length with advancing gestational age $^{3}$. On ultrasonography the sacrum is in the process ossification by 15 weeks of gestation ${ }^{5}$. In the second trimester, lordosis appear between the sacrum and the fifth lumbar vertebra and assist in more precise measurement.

The fetal sacrum is well seen in longitudinal section of caudal fetal spine as ossified last five vertebral bodies. The coccyx ossified after birth so it is sacrum which represents these caudal most vertebral bodies. In the third trimester, the sacrum is easier to measure and coccyx is different in echogenicity from that of ossified sacrum. 
2 did a cross sectional study on 2184 pregnant woman. All of the reviewed woman had uncomplicated singleton pregnancies without known structural and chromosomal fetal abnormality. They established a significant linear relationship between the fetal sacral length and the gestational age $\left(\mathrm{r}=0.98, \mathrm{R}^{2}=0.96\right)$. They also showed statistically significant correlation with $\quad \mathrm{BPD}(\mathrm{r}=0.68, \quad \mathrm{P}=0.001), \quad$ head circumference $(r=0.590, \quad \mathrm{P}=0.001) \quad \&$ $\mathrm{FL}(\mathrm{r}=0.719, \mathrm{P}=0.001)$.

This cross sectional study was done with an objective to find out correlation between sonographically measured fetal sacral lengths with gestational age.

The study included 400 healthy women and in this current study it was observed that the mean gestational age was 27.52 with standard deviation of mean $( \pm \mathrm{SD})$ was \pm 7.28 weeks and gestational age ranging from 15 to 40 weeks, which is consistent with ${ }^{2,3}$ observed gestational age range from 15 to 41 weeks.

Strong positive correlation was found between the gestational age measured from LMP and the fetal sacral length in $\mathrm{mm}$ measured by ultrasonography. The values of
Pearson's correlation coefficient was 0.998 , which was significant $(\mathrm{p}<0.001)$. Therefore, there was linear positive correlation between gestational age according to LMP in Bangladeshi subject and fetal sacral length measured in $\mathrm{mm}$ measured by ultrasonography. A regression analysis was also done. The coefficient of determination $\left(R^{2}\right)$ was found $99 \%$. From the above observation the null hypothesis of the present study was rejected and the alternative hypothesis was accepted.

\section{Conclusion}

This cross sectional study was done to find out the correlation between gestational ages with fetal sacral length measured by ultrasonography. This study result showed that fetal sacral length (FSL) is a reliable and valid method for assessing fetal age in a normal pregnancy.

In this study it is observed that there is significant correlation of fetal sacral length with estimated fetal age so, it can be concluded in the present study that fetal sacral length(FSL) can be used as a parameter for estimation of gestational age. 


\section{Reference.}

1.Peleg, D, Kennedy, CM and Hunter, SK 1998, intrauterine growth restriction; Identification and management, American Family Physicians, vol. 58, no.

2, pp. 453

2. Ozat M, et al: The significance of fetal sacral length in ultrasonographic assessment of gestational age. Arch GynecolObset(2011) 283: 999-1004.

3. Sherer DM, Abramowicz JS, Plessinger MA, Woods JR (1993). Fetal sacral length in ultrasonographic assessment of gestational age.Am $\mathrm{J}$ ObstetGynecol 168(2); 626-633.

4. Willocks, J, Donald, I, Duggen, T, Day, $\mathrm{N}$ et al. 1964, Fetalcephalometery by ultrasound, British Journal of Obstetrics and Gynaecology, vol. 71, pp.11-20.

5. Willium PL, Bannister LH, Berry M, Collins P, Dyson M, Dussek JE, Ferguson MWJ.Gray's Anatomy. $38^{\text {th }}$ ed. New York: ELBS with Churchill livingstone, 1995; pp 1815-1826. 